\title{
COMPORTAMIENTO DE VARIABLES PREDICTIVAS DE EXACERBACIONES DE LA EPOC EN EL HOSPITAL NEUMOLÓGICO DE CUBA
}

\author{
BEHAVIOR OF PREDICTIVE VARIABLES OF EXACERBATIONS OF THE COPD IN THE NEUMOLOGICAL \\ HOSPITAL OF CUBA.
}

\author{
Yusbiel José León Valdivies; ${ }^{1}$ Reinaldo B. Sánchez de la Osa; ${ }^{2}$ Eberto García Silvera; ${ }^{3}$ Delfina \\ Machado Molina; ${ }^{4}$ Liliana Oses Herrera. ${ }^{5}$
}

\begin{abstract}
RESUMEN
La utilización de variables predictoras de exacerbaciones de la EPOC no es una práctica generalizada en nuestro medio, por lo que no podemos caracterizar los pacientes exacerbadores ni diseñar estrategias para su manejo integral. Se realizó un estudio descriptivo prospectivo correlacionar en pacientes con diagnóstico de EPOC del Hospital Neumológico de Cuba, con el objetivo de determinar la asociación entre variables clínicas, funcionales e imagenológicas y la frecuencia de exacerbaciones al año. La población estuvo constituida por pacientes con diagnóstico clínico de EPOC y la muestra por aquellos pacientes con diagnóstico confirmado que cumplieran los criterios de inclusión. La correlación entre las variables se realizó mediante el Coeficiente de Correlación de Pearson con un intervalo de Confianza del $95 \%$ y la prueba $t$ de student con un nivel de significación (p) menor de 0.05 .El $81.82 \%$ de los pacientes muy graves son agudizadores con enfisema. El $75 \%$ de los pacientes con índice de la arteria pulmonar/ aorta tienen más de dos exacerbaciones al año. El $84.61 \%$ de los pacientes agudizadores presentaron grado cuatro de la disnea. La presión media de la arteria pulmonar junto al VEF1 constituyeron los mejores predictores de exacerbaciones en el grupo de pacientes estudiados.
\end{abstract}

Palabras claves: EPOC, exacerbaciones, presión media de la arteria pulmonar, variables predictivas, VEF1.

\begin{abstract}
The use of predictive variables of exacerbations of the COPD is not a practice generalized in our environment, for what we cannot characterize the exacerbating patient neither to design strategies for its integral handling. There was carried out a prospective descriptive study to correlate in patient with diagnosis of COPD from the Neumologic Hospital of Cuba, with the objective of determining the association between
\end{abstract}

1. Servicio de Neumotisiología. Hospital Neumológico Benéfico Jurídico. ylv87@nauta.cu

2. $\quad$ Servicio de Imagenología. Hospital Neumológico Benéfico Jurídico.

3. Servicio de Neumotisiología. Hospital Neumológico Benéfico Jurídico.

4. Servicio de Neumotisiología. Hospital Neumológico Benéfico Jurídico.

5. Residente de primer año de Estomatología General Integral. Facultad de Estomatología de Cienfuegos. 
clinical, functional variables and imagenological and the exacerbations frequency a year. The population was constituted for patients with clinical diagnosis of COPD and the sample for those patients with confirmed diagnosis that they completed the inclusion approaches. The correlation among the variables was carried out by means of the Coefficient of Correlation of Pearson with an interval of Trust of $95 \%$ and the test $t$ student with a significance level $(p)$ smaller than $0.05 .81 .82 \%$ of the very serious patients are exacerbating with emphysema. $75 \%$ of the patients with index of the lung artery / aorta have more than two exacerbations a year. $84.61 \%$ of the patient exacerbating presented degree four of the dyspnea. The half pressure of the lung artery next to the VEF1 constituted the best exacerbations predictors in the group of studied patients.

Key words: COPD, exacerbations, half pressure of the lung artery, predictive variables, VEF1

\section{Introducción}

La enfermedad pulmonar obstructiva crónica (EPOC) es una de las enfermedades crónicas que en los últimos decenios ha ido incrementando su incidencia, con un alto poder incapacitante y aumento de la morbimortalidad. La EPOC es una enfermedad pulmonar prevenible y tratable caracterizada por la limitación crónica al flujo aéreo, la cual no es totalmente reversible. Clínicamente se presenta con tos, expectoración y disnea de esfuerzo, y está asociada principalmente al humo de tabaco, a la exposición continua a los productos de la combustión de la biomasa y al déficit homocigoto de alfa-1-antitripsina ( $\alpha-1-A T T) .{ }^{(1)}$

La obstrucción se define mediante la espirometría a través de la determinación de la relación entre el volumen espiratorio forzado en el primer segundo (VEF1) y la capacidad vital forzada (CVF) (relación VEF1/CVF) menor de 0.7 (VEF1/CVF<0.7) y se clasifica después de la administración de un broncodilatador adrenérgico de acción corta salbutamol o terbutalina-, teniendo en cuenta los valores porcentuales del VEF1 postbroncodilatador con respecto al predicho. ${ }^{(2)}$

La denominación de fenotipo clínico se ha venido utilizando desde el $2010^{(3)}$ por un grupo de expertos internacionales, los que lo han definido como "aquellos atributos de la enfermedad que, solos o combinados, describen las diferencias entre individuos con EPOC en relación con parámetros que tienen significado clínico (síntomas, agudizaciones al año, respuesta al tratamiento, velocidad de progresión de la enfermedad)". (4) Esta definición permite identificar grupos de pacientes con diferente mortalidad a mediano y largo plazo. ${ }^{(5,6)}$ Diversos estudios recientes apoyan, la identificación de 4 fenotipos clínicos de la EPOC: fenotipo no agudizador, fenotipo mixto EPOC-asma, fenotipo agudizador con enfisema, fenotipo agudizador con bronquitis crónica. (3)

La agudización o exacerbación de la EPOC es un evento agudo caracterizado por el empeoramiento mantenido de los síntomas respiratorios de paciente, que va más allá de las variaciones diarias y un cambio en la conducta terapéutica. (7) Las exacerbaciones son eventos de consideración importante ya que: afectan negativamente la calidad de vida de los pacientes, ${ }^{(8,9)}$ se asocian con la pérdida acelerada de la función pulmonar, ${ }^{(10-12)}$ son relacionadas con aumento significativo de la mortalidad particularmente en los casos que requieren hospitalización, tienen un elevado costo socioeconómico (13)

Resulta difícil prevenir la exacerbación de la EPOC, porque no existen pródromos antes de su aparición o éstos son demasiado cortos como para detectarlos a tiempo. Considerando la exacerbación como un episodio que deteriora la función a largo plazo y que en sí misma puede suponer un deterioro progresivo de función si las 
exacerbaciones se repiten, cualquier factor modificador de éstas y de su frecuencia debería tener un efecto añadido en la reducción de la pérdida de función, la calidad de vida y, quizá, también de la mortalidad. Diversos estudios han demostrado que la media de agudizaciones de la EPOC es de 2 agudizaciones por paciente al año, y una de cada 6 exacerbaciones requerirá ingreso hospitalario, ${ }^{(14)}$ esto desencadena modificaciones pertinentes de tratamiento, generando un importante consumo de recursos sanitarios.

En nuestro país, según el Anuario Estadístico de

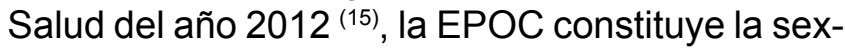
ta causa de muerte y es La Habana la que mayor mortalidad presenta con 885 defunciones a cusa de la EPOC, predominando en el sexo masculino y reportándose una tasa de años de vida potencialmente perdidos (AVPP) de 1.6 por 1000 habitantes, en pacientes de 1-74 años. De tal modo teniendo en cuenta todas las consecuencias sobre la salud humana y los costos económicos producidos por la enfermedad, es de vital importancia poder identificar a los pacientes con riesgos para estos eventos y/o contar con herramientas predictoras de dichas exacerbaciones.

La utilización de variables que pueden utilizarse como predictoras de exacerbaciones en la evaluación de los pacientes con EPOC, no es una práctica generalizada en nuestro medio, por lo que no podemos caracterizar a los pacientes exacerbadores con EPOC, ni diseñar sobre la base de esa caracterización, las estrategias pertinentes para el manejo integral de los mismos y la adquisición de los recursos necesarios para llevarlas a cabo.

\section{Material y Método}

Se realizó un estudio descriptivo prospectivo correlacionar en pacientes con EPOC del Hospital Neumológico de Cuba. La población estuvo constituida por pacientes con diagnóstico clínico de EPOC. La muestra quedó conformada por pacientes con diagnóstico confirmado de EPOC que cumplieran los criterios de inclusión: pacientes con diagnóstico confirmado de EPOC moderada, grave y muy grave según el Índice de BODE; pacientes fumadores o ex-fumadores y que sus condiciones clínicas les permitiera participar en el estudio con previo consentimiento. Una vez seleccionados los pacientes se les realizó un seguimiento trimestral y una observación estricta durante los períodos de descompensación. Posteriormente se determinó el grado de disnea según la escala modificada de la MRC y se realizó el cálculo del Índice de Masa Corporal. Las pruebas funcionales ventilatorias fueron realizadas en el laboratorio de nuestro centro con un equipo Spirometer DATOSPIR mod. 120B (Sibelmed®). EI Test de la Marcha de los seis minutos fue realizado en nuestro centro por el técnico especializado. La evaluación del estado de gravedad de la enfermedad se realizó teniendo en cuenta los datos clínicos y la aplicación del Índice de BODE. Se midieron las dimensiones de la arteria pulmonar a nivel de su bifurcación y de la arteria aorta en el mismo corte tomando un eje látero-lateral, mediante la tomografía computarizada de alta resolución sin contraste en un tomógrafo 16 Emotion. Se calculó la presión media de la arteria pulmonar basada en la velocidad pico del flujo de regurgitación tricuspídea; a través de la ecuación simplificada de Bernoulli se calculó la presión sistólica de la arteria pulmonar y posteriormente se calculó la presión media de la arteria pulmonar a partir de la presión sistólica de la arteria pulmonares utilizando la siguiente ecuación presión media de la arteria pulmonar $=0,61$ xpresión sistólica de la AP +2 $\mathrm{mmHg}$. Los datos se vaciaron en la planilla individual y posteriormente pasaron a la base de datos en la aplicación Microsoft Excel 2010 de la suite de Microsoft Office. El análisis estadístico se realizó en el programa EPIDAT versión 3.1; utilizamos como medidas de resumen los valores absolutos y los porcientos. La correlación entre las variables se realizó mediante el Coeficiente de Pearson ( $r$ ) y se utilizaron para el análisis de significación estadística el Intervalo de Confianza (IC) del 95\% y la prueba t de student con un nivel de significación (p) menor de $0.05(p<0.05)$.

\section{Resultados}

Se realizaron estudios clínicos, funcionales e imagenológicos a 30 pacientes con EPOC seguidos por consulta externa, con previa firma de la carta de consentimiento informado avalado por el Comité de Ética del Hospital Neumológico de Cuba. Del total de pacientes no agudizadores 7 (58.33\%) 
fueron clasificados como EPOC moderada según el índice de BODE. De los agudizadores con enfisema $9(81.82 \%)$ fueron clasificados como muy graves y $6(85.71 \%)$ pacientes del total de enfermos con fenotipo agudizador con bronquitis crónica fueron clasificados como graves.

Tabla 1. Relación entre el grado de disnea, el Índice de masa corporal y el número de exacerbaciones al año. $(n=30)$

\begin{tabular}{llll} 
& \multicolumn{3}{l}{ Frecuencia de exacerbaciones/año } \\
Variables predictoras & $\begin{array}{l}<\text { exac.l } \\
\text { año }\end{array}$ & $\begin{array}{l}<\text { exac./ } \\
\text { año }\end{array}$ & Total \\
Grado de disnea & No (\%) & No $(\%)$ & No $(\%)$ \\
Grado 1 & $5(83.33)$ & $1(16.66)$ & $6(20)$ \\
Grado 2 & $5(45.45)$ & $6(54.54)$ & $11(36.60)$ \\
Grado 3 & $2(15.38)$ & $11(84.61)$ & $13(43.30)$ \\
IMC & & & \\
$\leq 18.5$ & $1(16.66)$ & $5(83.33)$ & $6(20)$ \\
$18.6-24.9$ & $9(42.85)$ & $12(57.14)$ & $21(70)$ \\
$25-29.9$ & $2(66.66)$ & $1(33.33)$ & $3(10)$
\end{tabular}

La relación entre el grado de disnea y la frecuencia de exacerbaciones al año se muestra en la tabla 1 [ $r=0.42$ (IC 95\% 0.07-0.67; p= 0.02)]; el mayor número de pacientes se encuentran en los grados dos y tres de la disnea para un $41.66 \%$ respectivamente y $11(61.2 \%)$ de los pacientes con dos o más exacerbaciones al año, presentaron grado de disnea 4. La relación entre el índice de masa corporal y la frecuencia de exacerbaciones/año se describe en la tabla 1 [ $r=-0.27$ (IC $95 \%-0.10-0.57 ; p=0.14)]$.

La relación entre el VEF1 y la frecuencia de exacerbaciones al año se describe en la tabla $2[\mathrm{r}=$ -0.48 (IC 95\% $0.14-0.71 ; p=0.007)] ; 10$ (58.82\%) de los pacientes con dos o más exacerbaciones al año presentan valores del VEF1 entre $30 \% \leq y$ $<50 \%$ y los tres pacientes estudiados con valores del VEF1<30 presentan 2 o más exacerbaciones al año.
Tabla 2. Relación entre el VEF1 (\%), la distancia recorrida en el test de la marcha y el número de exacerbaciones al año. $(n=30)$

\begin{tabular}{llll} 
Variables predictoras & \multicolumn{3}{l}{ Frecuencia de exacerbaciones/año } \\
& $\begin{array}{l}<2 \text { exac.l } \\
\text { año }\end{array}$ & $\begin{array}{l}<\text { exac.l } \\
\text { año }\end{array}$ & Total \\
\hline VEF1 (\%) & No $(\%)$ & No $(\%)$ & No $(\%)$ \\
$50 \leq-<80$ & $5(50)$ & $5(50)$ & $10(33.3)$ \\
$30 \leq-<50$ & $7(41.17)$ & $10(58.82)$ & $17(56.6)$ \\
$<30$ & - & $3(100)$ & $3(10)$ \\
Distancia recorrida & & & \\
$\geq 350$ & $3(60)$ & $2(40)$ & $5(16.6)$ \\
$250-349$ & $6(50)$ & $6(50)$ & $12(40)$ \\
$150-249$ & $3(30)$ & $7(70)$ & $10(33.3)$ \\
$\leq 149$ & - & $3(100)$ & $3(10)$
\end{tabular}

En la tabla 2 muestra además la relación entre la distancia en metros recorrida durante el Test de Marcha de los seis minutos y la frecuencia de exacerbaciones al año. [ $r=-0.35$ (IC 95\% $-0.63-$ $0.012 ; p=0.05)$.

Tabla 3. Relación entre el índice arteria pulmonar/arteria aorta, la presión de la arteria pulmonar y la frecuencia de exacerbaciones al año. $(n=30)$

\begin{tabular}{|c|c|c|c|}
\hline \multirow[t]{2}{*}{ Variables predictoras } & \multicolumn{3}{|c|}{ Frecuencia de exacerbaciones/año } \\
\hline & $\begin{array}{l}<2 \text { exac.l } \\
\text { año }\end{array}$ & $\begin{array}{l}<2 \text { exac.I } \\
\text { año }\end{array}$ & Total \\
\hline Índice Ap/A & No $(\%)$ & No $(\%)$ & No $(\%)$ \\
\hline$A P: A \leq 1$ & $8(57.14)$ & $6(42.85)$ & $14(100)$ \\
\hline$A P: A>1$ & $4(25)$ & $12(75)$ & $16(100)$ \\
\hline \multicolumn{4}{|c|}{ Presión de la arteria Pulmonar } \\
\hline$<25$ & $11(52.38)$ & $10(47.61)$ & $21(70)$ \\
\hline$\geq 25-\leq 30$ & $1(50)$ & $1(50)$ & $2(6.66)$ \\
\hline$>30-<40$ & - & $4(100)$ & 4(13.33) \\
\hline$>40$ & - & $3(100)$ & $3(10)$ \\
\hline
\end{tabular}

La relación entre el índice de la arteria pulmonar/ arteria aorta y la frecuencia de exacerbaciones al año se expone en la tabla 3 [ $r=0.18$ (IC 95\% -0.19 - 0.50; $p=0.34)$ ]; de 14 pacientes con un índice AP: $A \leq 1,8$ (57.14\%) presentaban menos de dos exacerbaciones al año y en el grupo de 12 pa- 
cientes con un índice AP: A>1 el 75\% presenta dos o más exacerbaciones al año.

La tabla 3 muestra también la relación [ $\mathrm{r}=0.52$ (IC $95 \% 0.19-0.74 ; p=0.003)$ ] que existe entre los valores de presión media de la arteria pulmonar y el número de exacerbaciones al año; de un total de 21 pacientes con una presión media de la arteria pulmonar menor de $25 \mathrm{mmHg}, 11(52.38 \%)$ presentaron menos de dos exacerbaciones al año y en los grupos de pacientes con presión media de la arteria pulmonar $>30-<40 \mathrm{mmHg}$ y $>40$ de $\mathrm{mmHg}$ respectivamente, el $100 \%$ de pacientes presentan dos o más exacerbaciones al año.

Figura 1. Gráfica de dispersión que muestra la relación entre los valores del VEF1 (\%) y los valores de la presión media de la arteria pulmonar. $(r=-0.528, p=0.009)$

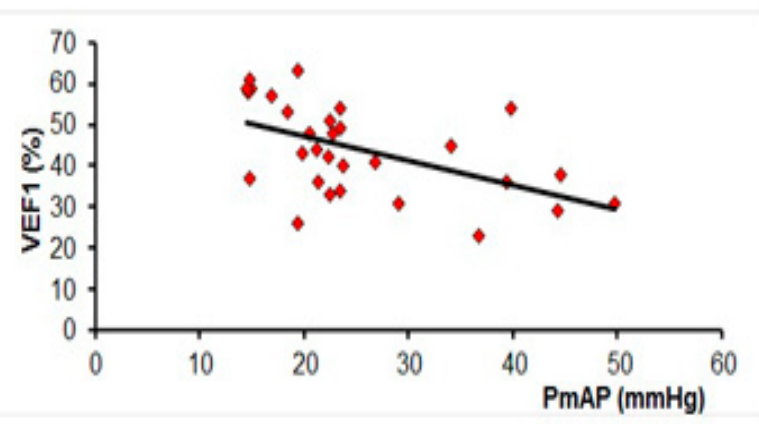

La relación entre el VEF1 y la presión media de la arteria pulmonar como predictoras de las exacerbaciones de la EPOC aparece en la figura 1. Del total de los 21 pacientes con cifras de presión media de la arteria pulmonar menor de $25 \mathrm{mmHg}, 11$ presentan valores de VEF1 $30 \% \geq y<50 \%$. Por otro lado el $100 \%$ de los pacientes que presentan cifras de presión media de la arteria pulmonar 25$30 \mathrm{mmHg}$ presentan valores de VEF1 $30 \% \geq \mathrm{y}<$ $50 \%$ y del total de pacientes con cifras de presión media de la arteria pulmonar de $>30-40 \mathrm{mmHg}$ y el de $>40 \mathrm{mmHg}$, el $50.00 \%$ y $66.66 \%$ respectivamente presentan valores de VEF1 $30 \% \geq \mathrm{y}<$ $50 \%$.

La actualización de la Carga Mundial de Enfermedades 2010 indica un incremento del número de personas con EPOC desde la anterior cifra en 1990 de 210 millones de personas con EPOC en todo el mundo, a la actual de 328.615 .000 de per- sonas con EPOC (168 millones en varones y 160 millones en mujeres). (16)Del total de los costos, causados por la EPOC a nivel mundial, las exacerbaciones explican $45 \%$ - $75 \%$ teniendo en cuenta gastos directos de atención sanitaria. (17) En este estudio se incluyeron pacientes con diagnóstico clínico de EPOC, los cuales presentaron distintos niveles de gravedad establecidos a partir del índice de BODE, además se evaluaron variables predictivas de las exacerbaciones de la enfermedad. Se evaluaron a treinta pacientes clínica, funcional e imagenológicamente de los cuales los que presentaron fenotipos clínicos agudizadores, ya sea con bronquitis crónica o con enfisema, presentaron peores estados de gravedad. Los pacientes agudizadores presentaron grados mayores de disnea y aquellos con un IMC menor presentaron más agudizaciones al año, comportándose el número de exacerbaciones al año inversamente proporcional al IMC. La mayoría de los pacientes con bajos valores de VEF1 presentaron dos o más exacerbaciones al año y el mayor número de aquellos que presentan menos metros recorridos durante el Test de la Marcha de los seis minutos tuvieron dos o más exacerbaciones al año. Los mayor parte de los pacientes con un índice arteria pulmonar/aorta mayor de uno presentaron dos o más exacerbaciones al año. El mayor número de pacientes con hipertensión pulmonar presentan dos o más exacerbaciones al año. Los pacientes con la presión media de la arteria pulmonar elevada presentan peores valores del VEF1. Creemos que es de vital importancia la realización de estudios que impliquen las variables predictivas de las exacerbaciones de la EPOC lo que permitiría planificar programas más integral e individualizados a cada paciente exacerbador, mejorando la calidad de vida y además una mejor planificación económica de los servicios de salud para enfrentar los altos costos de la enfermedad. Consideramos que aunque la muestra es limitada, es fundamental para continuar realizando investigaciones para encontrar valores predictivos que puedan ser de utilidad clínica.

\section{Referencias Bibliográficas}

1. Celli BR, MacNee W. Standards for the diag- 
nosis and treatment of patients with COPD: a summary of the ATS/ERS position paper. EurRespir J. 2004;23(6):932-946.

2. Global Strategy for Diagnosis, Management, and Prevention of COPD (Guidelines) Updated 2010. http://goldcopd.com/GuidelinesResources.

3. Miravitllesa M, Soler-Cataluñab JJ, Calle M, et al. Guía española de la EPOC (GesEPOC). Actualización 2014. ArchBronconeumol. 2014;50:1-16

4. Han MK, Agusti A, Calverley PM, Celli BR, Criner G, Curtis JL, et al. Chronic obstructive pulmonary disease phenotypes. The future of COPD. Am J RespirCric Care Med. 2010; 182:598-604.

5. Burgel P, Roche N, Paillasseur J, Tillie-Leblond I, Chanez P, Escamilla R, et al; INITIATIVES BPCO Scientific Committee. Clinical COPD phenotypes identified by cluster analysis: validation with mortality. EurRespirJ. 2012;40:495.

6. García-Aymerich J, Gómez FP, Benet M, Farrero E, Basagaña X, Gayete A, et al; PAC-COPD Study Group. Identification and prospective validation of clinically relevant chronic obstructive pulmonary disease (COPD) phenotypes. Thorax. 2011;66:430-7.

7. Celli BR, Barnes PJ. Exacerbations of chronic obstructive pulmonary diseas. EurRespir J 2007; 29: 1224-38.

8. Spencer S, Calverley PM, Burges PS. Impact of preveting exacerbations on deterioration of health status in COPD. EurRespir J 2004; 23: 698.

9. Kessler R, Stahl E, Vogelmeier, et al. Patient understanding, detection, and experience of COPD exacerbations: an observational, interview- based study. Chest 2006; 130:133-42.
10. Grupo de Trabajo de GesEPOC. Guía de Práctica Clínica para el Diagnóstico y Tratamiento de Pacientes con Enfermedad Pulmonar Obstructiva Crónica (EPOC) - Guía Española de la EPOC (GesEPOC) ArchBronconeumol. 2012;48 (Supl 1):2-58

11. Donaldson GC, Seemungal TA, Bhowmik A, Wedzicha JA. Relationship between exacerbation frequency and lung function decline in chronic obstructive pulmonary disease. Thorax 2002;57:847-52.

12. Agusti AG, Noguera A, Sauleda J, Sala F, Pons $J$, Busquets $X$. Systemic effects of chronic obstructive pulmonary disease. EurRespira J 2003; 21(2):347.

13. Traves SL, Culpitt SV, Russell RE, Barnes PJ, Donelly LE. Increased level of chemokinesGROalpha and MCP-1 insputum samples from patients with COPD. Thorax. 2002;57:590-5.

14. Tálamo C, de Oca MM, Halbert R, Perez-Padilla $R$, Jardim JR, Muiño A, et al. Diagnostic labelling of chronic obstructive pulmonary disease in five Latin American cities. Chest. 2007;131:60-7.

15. Centro de Estudios de la Población y DesarroIlo. Anuario demográfico de Cuba 2012. Oficina Nacional de Estadísticas; 2012. Disponible en http://files.sld. cu/dne/files/2013/04/anuario_2012.pdf.

16. López Varela M.V. y Montes de Oca M. Variabilidad en la EPOC, una visión a través del estudio PALATINO. ArchBronconeumol. 2012; 48 (4):105.

17. Toy EL, Gallagher KF, Stanley EL, Swensen $A R$, Duh MS. The eco-nomic impact of exacerbations of chronic obstructive pulmonary disease and exacerbation definition: a review. COPD. 2010;7(3):214-228. 